Kubandt, Melanie

\title{
Weiblichkeit als Normalitätsfolie in der Kindertageseinrichtung. Empirische Perspektiven auf die gendered occupation der pädagogischen Fachkraft
} Langer, Antje [Hrsg.]; Mahs, Claudia [Hrsg.]; Rendtorff, Barbara [Hrsg.]: Weiblichkeit - Ansätze zur Theoretisierung. Opladen; Berlin; Toronto : Verlag Barbara Budrich 2018, S. 103-112. - (Jahrbuch Frauenund Geschlechterforschung in der Erziehungswissenschaft; 14)

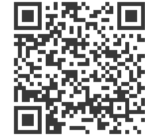

\section{Quellenangabe/ Reference:}

Kubandt, Melanie: Weiblichkeit als Normalitätsfolie in der Kindertageseinrichtung. Empirische Perspektiven auf die gendered occupation der pädagogischen Fachkraft - In: Langer, Antje [Hrsg.]; Mahs, Claudia [Hrsg.]; Rendtorff, Barbara [Hrsg.]: Weiblichkeit - Ansätze zur Theoretisierung. Opladen; Berlin; Toronto : Verlag Barbara Budrich 2018, S. 103-112 - URN: urn:nbn:de:0111-pedocs-190836 DOI: 10.25656/01:19083

\section{https://nbn-resolving.org/urn:nbn:de:0111-pedocs-190836} https://doi.org/10.25656/01:19083

in Kooperation mit / in cooperation with:

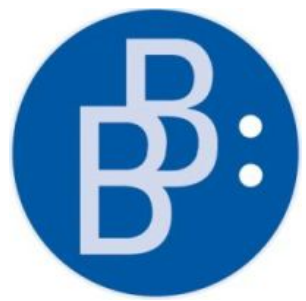

https://www.budrich.de

\section{Nutzungsbedingungen}

Dieses Dokument steht unter folgender Creative Commons-Lizenz: http://creativecommons.org/licenses/by-sa/4.0/deed.de - Sie dürfen das Werk bzw. den Inhalt vervielfältigen, verbreiten und öffentlich zugänglich machen sowie Abwandlungen und Bearbeitungen des Werkes bzw. Inhaltes anfertigen, solange sie den Namen des Autors/Rechteinhabers in der von ihm festgelegten Weise nennen und die daraufhin neu entstandenen Werke bzw. Inhalte nur unter Verwendung von Lizenzbedingungen weitergeben, die mit denen dieses Lizenzvertrags identisch, vergleichbar oder kompatibel sind.

Mit der Verwendung dieses Dokuments erkennen Sie die Nutzungsbedingungen an.

\section{Terms of use}

This document is published under following Creative Commons-License: http://creativecommons.org/licenses/by-sa/4.0/deed.en - You may copy distribute and transmit, adapt or exhibit the work or its contents in public and alter, transform, or change this work as long as you attribute the work in the manner specified by the author or licensor. New resulting works or contents must be distributed pursuant to this license or an identical or comparable license.

By using this particular document, you accept the above-stated conditions of use.

\section{Kontakt / Contact:}

peDOcs

DIPF | Leibniz-Institut für Bildungsforschung und Bildungsinformation Informationszentrum (IZ) Bildung

E-Mail: pedocs@dipf.de

Internet: www.pedocs.de

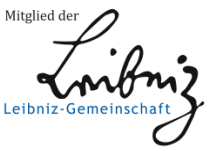


Antje Langer

Claudia Mahs

Barbara Rendtorff (Hrsg.)

\section{$14 / 2018$}

Jahrbuch Frauen- und Geschlechterforschung in der Erziehungswissenschaft Weiblichkeit - Ansätze zur Theoretisierung 
Jahrbuch

Frauen- und Geschlechterforschung

in der Erziehungswissenschaft

Folge 14/2018

Redaktion

Jürgen Budde

Marita Kampshoff

Astrid Messerschmidt

\section{Beirat}

Birgit Althans, Sabine Andresen, Robert Baar, Rita Casale, Bettina Dausien, Isabell Diehm, Hannelore FaulstichWieland, Susann Fegter, Edgar Forster, Edith Glaser, Jutta Hartmann, Carola Iller, Margret Kraul, Andrea Liesner, Martin Lücke, Susanne Maurer, Vera Moser, Inga Pinhard, Melanie Plößer, Annedore Prengel, Barbara Rendtorff, Anita Thaler, Christine Thon, Katharina Walgenbach 


\author{
Antje Langer \\ Claudia Mahs \\ Barbara Rendtorff (Hrsg.)
}

\title{
Weiblichkeit - Ansätze zur Theoretisierung
}

Verlag Barbara Budrich

Opladen • Berlin • Toronto 2018 
Bibliografische Information der Deutschen Nationalbibliothek

Die Deutsche Nationalbibliothek verzeichnet diese Publikation in der Deutschen

Nationalbibliografie; detaillierte bibliografische Daten sind im Internet über

http://dnb.d-nb.de abrufbar.

(C) 2018 Dieses Werk ist bei Verlag Barbara Budrich erschienen und steht unter folgender Creative Commons Lizenz:

https://creativecommons.org/licenses/by-sa/4.0/

Verbreitung, Speicherung, Vervielfältigung und Veränderung unter Angabe zu

UrheberInnen, Rechten, Änderungen und der verwendeten Lizenz erlaubt.

\section{(c) (i) (2)}

Dieses Buch steht im Open-Access-Bereich der Verlagsseite zum kostenlosen Download bereit (https://doi.org/10.3224/jfgfe.v14i1).

Eine kostenpflichtige Druckversion (Printing on Demand) kann über den Verlag bezogen werden. Die Seitenzahlen in der Druck- und Onlineversion sind identisch.

$$
\begin{array}{ll}
\text { ISBN } & 978-3-8474-2194-8 \text { (Paperback) } \\
\text { eISBN } & 978-3-8474-1220-5 \text { (eBook) } \\
\text { DOI } & 10.3224 / \text { jfgfe.v14i1 }
\end{array}
$$

Umschlaggestaltung: disegno visuelle kommunikation, Wuppertal - www.disenjo.de Typografisches Lektorat: Anja Borkman, Jena

Verlag Barbara Budrich, http://www.budrich-verlag.de 


\section{Inhalt}

\section{Thementeil}

Barbara Rendtorff \& Antje Langer

Einleitung....

Meike Sophia Baader

Von der Normalisierung zur De-Zentrierung nach 1968.

Mütterlichkeit, Weiblichkeit und Care in der Alten und in der Neuen

Frauenbewegung

Bastian Lasse

,Pioniergeist" im Worte. Weiblichkeitskonstruktion bei Frieda von

Bülow.

Anna Orlikowski

Weiblichkeit als Zweideutigkeit: Phänomenologische Zugänge im

Spannungsfeld zwischen Beschreibung und Konstituierung

Frauke Grenz

„Das muß ihr als Frau erstmal eine oder einer nachmachen!“ Sind Angela Merkel und Ségolène Royal Beispiele für hegemoniale Weiblichkeit?

Carrie Paechter

Hegemonic femininities in the classroom

Melanie Kubandt

Weiblichkeit als Normalitätsfolie in der Kindertageseinrichtung empirische Perspektiven auf die gendered occupation der pädagogischen Fachkraft 


\section{Offener Teil}

Arzu Çiçek \& Astrid Messerschmidt

Anfeindungen der Differenz. Nation und Geschlecht in der deutschen

Migrationsgesellschaft

Maya Halatcheva-Trapp

Rationalität und Relationen. Zum Verhältnis von Weiblichkeit und Mütterlichkeit aus wissenssoziologisch-diskursanalytischer

Perspektive

Autor innen 


\title{
Weiblichkeit als Normalitätsfolie in der Kindertageseinrichtung - empirische Perspektiven auf die gendered occupation der pädagogischen Fachkraft
}

\author{
Melanie Kubandt
}

\section{Hintergrund}

Der Beitrag knüpft an aktuelle Debatten zu Geschlecht in Kindertageseinrichtungen an und baut auf empirische Ergebnisse der ethnographischen Studie „Geschlechterdifferenzierung in der Kindertageseinrichtung“ auf, die im Alltag einer Kindertageseinrichtung Geschlechterkonstruktionen von Kindern, Fachkräften und Eltern untersucht hat. Auf Basis von Teilergebnissen zu Geschlechterkonstruktionen pädagogischer Fachkräfte werden im Kontext der gendered institution Kindertageseinrichtung empirische Perspektiven auf Weiblichkeit dargelegt, die den aktuell dominierenden Fokus auf Männlichkeit in Kindertageseinrichtungen ergänzen. Zudem wird Geschlecht als relevanter Faktor in Kindertageseinrichtungen diskutiert.

\section{Die Geschlechtszugehörigkeit pädagogischer Fachkräfte - zentrales Bestimmungsmerkmal in Kindertageseinrichtungen?!}

Der Erzieher*innenberuf gilt nach wie vor als typischer Frauenberuf (vgl. Hoffmann 2012, Metzinger 2013, Karsten 2017). Laut Rohrmann et al. (2010) sind Kindertageseinrichtungen in Deutschland „der Bereich der Kinder- und Jugendhilfe mit dem niedrigsten Männeranteil“" (ebd.: 2). Mit aktuell mehr als 90 Prozent Anteil an beschäftigten Frauen sind Kindertageseinrichtungen daher bis heute ein weiblich dominiertes Tätigkeitsfeld: ,child care is largely woman`s work" (Murray 1996: 368). Historisch lässt sich bei dem Berufsbild der Erzieher*in bei Fröbel eine eingeschränkte Vorstellung von Weiblichkeit bzw. deutliche Nähe zur Mutterschaft rekonstruieren, die das Muttersein in den Fokus stellt (Hoffmann 2012: 337). Heite (2009) und Ehrenspeck (2009) rekonstruieren am Beispiel geistiger Mütterlichkeit gar den zentralen Stellenwert der Geschlechtszugehörigkeit für die Professionalisierung. Kindertageseinrichtungen sind in diesem Sinne „gendered institutions“ 
(Acker 1992) und Fachkräfte gehören einer ,gendered occupation“ an: „Gendered occupations are those occupations that are structured on the assumption that they will be occupied predominantly by workers in a single sex category“" (Murray 1996: 371). An diesem ungleichen Geschlechterverhältnis setzt gleichstellungspolitisch die Forderung nach mehr Männern in Kitas an. Maßgeblich ist hier die bundesweite, 2014 abgeschlossene Initiative „MEHR Männer in Kitas“, die aus 16 Modellprojekten bestand, an denen ca. 1.300 Kindertageseinrichtungen in 13 Bundesländern beteiligt waren und die mit insgesamt etwa 13 Millionen Euro aus dem Europäischen Sozialfonds gefördert wurden (vgl. Cremers et al. 2012: 10). Hintergrund dieser Initiative ist u.a. die Annahme, dass Geschlechtergerechtigkeit in Kindertageseinrichtungen nur dann verwirklicht werden kann, wenn sowohl männliche als auch weibliche Vorbilder für Kinder vor Ort fachlich tätig sind (vgl. Cremers/Krabel 2012a). ${ }^{1}$

\section{Geschlecht und Profession(alisierung) - Studien zu pädagogischen Fachkräften}

Für Forschungsarbeiten zu Fachkräften und Geschlecht gilt aktuell, dass der empirische Blick primär auf Zweigeschlechtlichkeit und dann spezifisch auf männliche Fachkräfte gerichtet wird (vgl. Rose/Stibane 2013: 31), sodass diejenigen Personen, die bislang im Feld der Kindertageseinrichtungen tätig waren - also die über 90\% weiblichen Fachkräfte -, nur wenig empirische Berücksichtigung erhalten (vgl. May/Rose 2014: 14).

In einer qualitativen Studie von Buschmeyer (2013) wurde bspw. untersucht, wie Männer im Erzieher*innenberuf Männlichkeit herstellen und dabei rekonstruiert, dass Männlichkeit durch die Wahl eines weiblich konnotierten Berufs tendenziell in Frage gestellt werde, gleichzeitig jedoch an die Erzieher Erwartungen gestellt würden, die in enger Verbindung zu gesellschaftlichen Vorstellungen einer hegemonialen Männlichkeit stünden (vgl. ebd.: 273ff.). Auch Breitenbach et al. (2015) haben in Interviews rekonstruiert, dass Männlichkeitskonstruktionen als eine spezifische Herausforderung für Erzieher betrachtet werden können. Bei einem Vergleich haben sie festgestellt, dass weibliche Erzieherinnen ihr Geschlecht nicht als thematisierungswürdig erachten, während die Geschlechtszugehörigkeit von Männern explizit und implizit betont wurde (ebd.: 159). Brandes et al. (2016) haben hingegen untersucht, ob und inwiefern sich weibliche und männliche Fachkräfte in ihrem pädagogischen Verhalten gegenüber Mädchen und Jungen im Alter von drei

1 Für je kritische Perspektiven auf diese These siehe Rose (2012), Fegter (2013), Breitenbach et al. (2015) und Kubandt (2016). 
bis sechs Jahren unterscheiden. Zentraler Befund war, dass keine signifikanten Unterschiede im Hinblick auf den professionellen Umgang mit Kindern nachgewiesen werden konnten.

Im Rahmen der Forderung nach „Mehr Männern in Kitas“ kommt es dabei immer wieder verstärkt zu einer Diskussion der Thematik sexueller Missbrauch und Generalverdacht gegenüber Männern. Cremers und Krabel (2012b) sprechen gar davon, ,dass es geradezu unmöglich ist, sich mit dem Thema Männer in Kitas zu beschäftigen, ohne mit dem Generalverdacht gegenüber Männern konfrontiert zu werden“ (ebd.: 265). Von ähnlichen Erfahrungen berichtete bereits Murray (1996) in ihrer Studie: „Specifically, in many centers, men are more restricted in their freedom to touch, cuddle, nap, and change diapers for children" (ebd.: 378). Zudem würden männlichen Fachkräften niedere Motive unterstellt: „When men chose child care, their motives for making such a choice are questioned. [...] they are suspect just for doing their work" (ebd.: 377f.). Auch Aigner und Rohrmann (2012) kommen in ihrer Studie zu Erfahrungen von männlichen Erziehern zu dem Ergebnis, dass der Verdacht der Pädophilie zu einer Beschränkung der Erzieher in ihrem professionellen Handeln führt und sie vorsichtig agieren lässt.

\section{Die weiblichen Fachkräfte - Das unsichtbare und unschuldige Geschlecht?!}

Im Folgenden stehen Teilergebnisse aus der ethnographischen Studie „Geschlechterdifferenzierung in der Kindertageseinrichtung" (vgl. Kubandt 2016) im Fokus, deren zentrale Forschungsfrage lautete ,Wie wird Geschlecht von den Akteur*innen im Feld der Kindertageseinrichtung konstruiert und mit welchen Relevanzsetzungen wird Geschlecht verknüpft?" Die methodologische Forschungsfolie bildete der Ansatz des doing gender (vgl. Garfinkel 1967). Die Beobachtungsprotokolle wurden im Zeitraum von 14 Monaten mithilfe der teilnehmenden Beobachtung in einer niedersächsischen Kindertageseinrichtung generiert und dabei Geschlechterkonstruktionen von Fachkräften, Kindern und Eltern fokussiert.

Im Sinne eines reifizierungssensiblen Vorgehens wurden im Vorfeld keine spezifischen Situationen des Kindergartenalltags für die teilnehmende Beobachtung ausgewählt. Neben der teilnehmenden Beobachtung kamen entsprechend ethnographischer Prinzipien der methodischen Flexibilität und Offenheit im Forschungsprozess auch Videoaufnahmen, Artefakte und Dokumente zum Einsatz. Parallel zu den Erhebungen wurden zunächst erste Ausschnitte aus den Beobachtungsprotokollen sowie einzelne Videoszenen transkribiert und analysiert. Dabei dienten die Videotranskripte primär dazu, die Beobachtungsprotokolle zu ergänzen und zu verdichten, indem die video- 
basierten Transkriptionen sprachlicher Interaktionen in die Beobachtungsprotokolle integriert wurden, die dann letztlich den Schwerpunkt des auszuwertenden Datenkorpus bildeten. Die Analyse des Datenmaterials erfolgte in Anlehnung an die Grounded Theory (vgl. Strauss/Corbin 1996). Parallel zu fortlaufenden Kodierungen und Kategorisierungen wurde das vorstrukturierte Material mit einem sequenzanalytischen Vorgehen weiter aufbereitet, wie es u.a. im integrativen Basisverfahren nach Kruse (2014) vorgenommen wird. Eine Orientierung für die Auswahl der Sequenzen bot dabei die thematische Relevanz im Hinblick auf die Forschungsfrage nach den Geschlechterkonstruktionen im Alltag der Kindertageseinrichtung.

Im Folgenden stehen nun einzelne Ergebnisse zu den Konstruktionen der Fachkräfte im Fokus, die zeigen, wie unterschiedlich Männlichkeit und Weiblichkeit im pädagogischen Alltag zum Thema werden. So dominierte die weibliche Geschlechtszugehörigkeit der Fachkräfte den pädagogischen Alltag in der Kindertageseinrichtung offenbar derart, dass Weiblichkeit völlig in den Hintergrund trat, da es die geschlechtliche Normalitätsfolie darstellte (vgl. Kubandt 2016: 202ff.). ${ }^{2}$ Denn Geschlecht war kein Thema, das von den weiblichen Fachkräften im pädagogischen Alltag mit ihrer eigenen Personengruppe verknüpft wurde. Vielmehr wurde ihrerseits wiederholt formuliert, dass Geschlecht nur auf Ebene der Kinder bedeutsam sei. Entsprechend hieß es ihrerseits in ähnlicher Weise wiederholt: „Nicht mich filmen, Melanie, die Kinder! Ich bin für dein Thema doch völlig uninteressant". Als jedoch zum Ende der Erhebungsphase ein männlicher Erzieher namens Tom als Vertretung in die Einrichtung kam, ergaben sich mehrfach Begegnungen mit weiblichen Fachkräften, die in dessen Anwesenheit einen geschlechtlich relevanten Aspekt für mich als Forscherin sahen und Interaktionen zwischen Tom und Kindern u.a. wie folgt kommentierten:

Natascha sagt lachend zu mir, auf Tom deutend: „Oh, interessantes Thema für dich! Besser könnte es nicht sein!“

Zudem wurde das Interesse der Kinder an Tom von den weiblichen Fachkräften stets am Faktor Geschlecht festgemacht. Das heißt, die Relevanz von Geschlecht wurde sowohl auf Ebene des Erziehers als auch auf Ebene der Kinder als Interpretationsfolie zur Erklärung kindlicher Spielpräferenzen angelegt. Dies zeigt sich exemplarisch am Beispiel eines Protokollausschnitts, in dem die Fachkraft Anna kurz nach meiner morgendlichen Ankunft in der Kita ungefragt erzählt, warum zu jenem Zeitpunkt lediglich eine Gruppe von Mädchen im Gruppenraum ist:

Anna: „Heute ist Tom da, und da sind die Jungen dann weg!“ Forscherin: „Ja? Ist das so?“ Anna: „Ja, total, fast wie im Klischee könnte man meinen. Die Mädchen fahren zwar auch voll auf ihn ab, aber schon vor allem die Jungen, wenn er da ist. Die sind dann nur auf ihn fixiert."

2 Hier zeigen sich Parallelen zur Studie von Breitenbach et al. (2015). 
Dabei variierte, ob die weiblichen Fachkräfte kollektiv Jungen oder Mädchen ein besonderes Interesse an Tom zuschrieben. Häufig folgten entsprechenden Kommentierungen Aussagen wie diese: „Musste an dich denken, Melanie, dass das ja wieder was für dich ist! “ (vgl. Kubandt 2016: 211ff.).

Offenbar war die Akteur*innengruppe der Fachkräfte so selbstverständlich mit der weiblichen Geschlechtszugehörigkeit verbunden, dass dieser Sachverhalt im Alltag der Kindertageseinrichtung aus dem Blick geriet. Erst mit der Anwesenheit von Tom trat durch die Verknüpfung männliche Geschlechtszugehörigkeit und Fachkraft eine Abweichung vom Normfall bzw. eine Gegenfolie des Gängigen auf, wodurch die Relevanz der weiblichen Geschlechtszugehörigkeit indirekt als das unsichtbare Geschlecht in der Kindertageseinrichtung sichtbar wurde.

Dass die weibliche Geschlechtszugehörigkeit auf Ebene der Erwachsenen im Feld der Kindertageseinrichtung die Normalitätsfolie darstellt, zeigte sich auch im Hinblick auf meine Person. Denn offenbar war ich als Frau per se vertrauensvoll und kompetent, denn bereits vom ersten Erhebungstag an durfte ich unbeaufsichtigt über lange Zeiträume mit den Kindern allein interagieren. So ereignete es sich des Öfteren, dass einzelne Fachkräfte lediglich kurz den Kopf in den Raum steckten, um nach an mich gerichteten Kommentaren wie ,aah, $d u$ bist ja $d a$ " sofort wieder zu gehen. Folglich legitimierte meine Anwesenheit bei den Kindern ihre Abwesenheit. Erfreut über das entgegengebrachte Vertrauen, gab es nichtsdestotrotz immer wieder Situationen, in denen ich verunsichert war, was im Umgang mit den Kindern legitim ist und was nicht. Hierzu ein Auszug eines Protokolls, das zu Beginn der Erhebung entstand:

Ich werde von dem Kindergartenkind Isabell geholt, weil ihrer Aussage nach keiner da ist, um dem Krippenkind Maximilian den Hintern abzuwischen. Sie findet keine Fachkraft, daraufhin gehe ich ebenfalls auf die Suche, weil ich davon ausgehe, nicht befugt zu sein, den Kindern beim Klogang zu helfen, da das ggf. zu intim ist. Das sage ich auch der Fachkraft, die ich finde, die das aber lachend abtut.

Die Ungewissheit, was im Feld erlaubt ist, tangiert u.a. die Frage nach dem angemessenen körperlichen Kontakt zu den Kindern. Die Reaktion auf meine Vermutung, dass ich den Kindern unter Umständen körperlich zu nah käme und dies daher nicht dürfe, zeigt, dass die Fachkraft diese Annahme nicht teilt. Ich hingegen thematisiere eine potenziell heikle Komponente im engen Körperkontakt mit den Kindern. Dieser Sachverhalt kennzeichnet auch eine andere Situation im Feld:

Wir sind jetzt allein im Raum. Melina (2,5 Jahre) legt sich neben mich und kuschelt sich an mich ran. Ich finde es angenehm und könnte glatt wegdösen. Wir liegen da so eine Weile, Melina kuschelt sich mal mehr mal weniger an mich. Die Kita- und Gruppenleitung Sabine kommt rein, ich frage sie „Darf ich das eigentlich, so mit Melina kuscheln?“ Sabine nickt und sagt „Ja, warum denn nicht?!“ In dem Moment streicht Melina unbeabsichtigt kurz 
über meine Brust. Sabine sieht das und sagt „Na ja, man kann und muss dann halt auch sagen, wenn einem was unangenehm ist. Das ist dann völlig ok."

Dieses Beispiel zeigt, dass ich von den Fachkräften vertrauensvoll und unbeaufsichtigt mit Kindern allein gelassen werde. Dabei ist körperliche Nähe zwischen den Kindern und mir offenbar aus Sicht der Leitung völlig unproblematisch, was ihre Antwort ,Ja, warum denn nicht?! “ auf meine diesbezügliche Frage verdeutlicht. Die Antwort suggeriert, dass es aus ihrer Sicht keinen Grund geben könne, der dagegen spreche. Interessant ist, dass Melinas unbeabsichtigtes Berühren meiner Brust Sabine offenbar veranlasst, ihre vorherige Antwort zu konkretisieren: „Na ja, man kann und muss dann halt auch sagen, wenn einem was unangenehm ist. Das ist dann völlig ok. " Demnach ist Körperkontakt mit den Kindern solange in Ordnung, wie keine Grenzen gesetzt werden, d.h. demzufolge per se zunächst zugelassen. Wie weit das gehen darf, muss dann offenbar individuell von den Beteiligten verhandelt werden. Nimmt man allerdings die potenzielle Gefahr ernst, dass in pädagogischen Settings körperliche Übergriffe auf Abhängige erfolgen können, müsste zumindest diskutiert werden, dass potenziellen Opfern bzw. gerade kleinen Kindern häufig nicht bewusst sein kann, dass ggf. Grenzen überschritten werden.

Ob Körperkontakt im Umgang mit den Kindern generell, d.h. geschlechtsunabhängig als unproblematisch angesehen wird, ist nicht rekonstruierbar. Meine weibliche Geschlechtszugehörigkeit kann zumindest eine Erklärung für das entgegengebrachte Vertrauen sein, da ich dem gleichen unsichtbaren Geschlecht bzw. der Normalitätsfolie des Feldes entspreche. Das kann auch ein Grund dafür sein, dass ich schnell akzeptiert werde, viele Freiheiten habe und sowohl von den Kindern als auch den Fachkräften wiederholt die Rolle als Ersatzkraft zugeschrieben bekomme. Deshalb scheint plausibel, dass das entgegengebrachte Vertrauen v.a. mit meiner weiblichen Geschlechtszugehörigkeit zu tun hat. Der Möglichkeit eines Generalverdachts im Hinblick auf männliche Personen steht also eine mir als Frau entgegengebrachte „pauschale Unschuldsvermutung“ (Kubandt 2016: 205) gegenüber. Die Unschuldsvermutung kann im Grunde auch auf weibliche Fachkräfte generell übertragen werden, denn der Generalverdacht scheint untrennbar mit der männlichen Geschlechtszugehörigkeit verknüpft. Daher wurde die weibliche Geschlechtszugehörigkeit der Fachkräfte nicht nur als das vermeintlich unsichtbare, sondern auch als das vermeintlich unschuldige Geschlecht in der Kindertageseinrichtung rekonstruiert (vgl. ebd.). 


\section{Männlichkeit versus Weiblichkeit-Zum Un/Sinn kollektivierender Zuschreibungen in der Kindertageseinrichtung}

Sowohl aktuelle Debatten als auch der untersuchte Alltag der Kindertageseinrichtung sind durch kollektivierende Gegenüberstellungen von Männlichkeit und Weiblichkeit geprägt, wobei Weiblichkeit nur indirekt zum Thema wird, da diese als Normalitätsfolie aus dem Blick gerät. Dagegen beinhaltet die Forderung nach mehr männlichen Fachkräften eine kollektiv unterstellte Positivmarkierung von Männlichkeit. Hierbei wird nicht ausreichend diskutiert, inwiefern eine Vorbildfunktion, die rein über Geschlechtszugehörigkeiten von Fachkräften definiert wird, tendenziell dazu beiträgt, Stereotype in den Kindertageseinrichtungen zu verstärken statt zu vermeiden. Rose (2012) und Fegter (2013) sprechen hier kritisch von einem, die Debatten dominierenden, sozialisationstheoretischen Argument, das die Anwesenheit von Männern im Feld der Tageseinrichtungen als einen größeren Erfahrungsspielraum im Hinblick auf geschlechtliche Rollenvorbilder für Kinder argumentiert. Damit wird indirekt unterstellt, dass ein erweiterter Erfahrungsspielraum folglich immer an Geschlechtszugehörigkeit geknüpft bzw. nicht unabhängig davon gedacht werden kann. Die weibliche Geschlechtszugehörigkeit der Fachkraft wird demzufolge gleichgesetzt mit einer Begrenzung als Rollenvorbild für den Erfahrungsspielraum von Kindern und somit negativ markiert. Hierbei werden Unterschiede innerhalb der Gruppe weiblicher Fachkräfte ausgeblendet und zwischen den Geschlechtern betont, sowie Männlichkeit an sich als Mehrwert zur Bereicherung des Feldes als Ergänzung des Weiblichen angesehen. Indem Männer in das Feld integriert werden, werden diese Begrenzungen aus Sicht zahlreicher Vertreter*innen entsprechender Initiativen vermeintlich aufgehoben. Es erscheint allerdings paradox, dass die Erweiterung geschlechtlicher Erfahrungsspielräume von Kindern erreicht werden soll, indem gleichzeitig unterschiedliche Erfahrungsspielräume als stets geschlechtlich bedingt, d.h. jeweils mit Männern und Frauen bzw. Mannsein und Frausein verknüpft werden.

Unabhängig davon, ob Geschlecht eher problematisiert, d.h. negativ markiert oder als produktives Merkmal von Fachkräften positiv markiert wird, orientieren sich die auf den ersten Blick konträren Ausrichtungen dabei vor allem an kollektivierenden, den Situationen und Kontexten vorgängigen Geschlechterbildern, die an Individuen und pädagogische Situationen herangetragen werden, ohne dass diese anhand einzelner Personen oder des pädagogischen Alltags selbst hinterfragt und/oder erweitert werden.

Während aktuell Männlichkeit den Fokus frühpädagogischer Debatten prägt, konnte in Abgrenzung des genannten Generalverdachts zudem eine pauschale Unschuldsvermutung gegenüber Weiblichkeit rekonstruiert wer- 
den. Da plausibel scheint, dass die Fachkräfte in anderen Kontexten durchaus auf Weiblichkeit rekurrieren, wird eine Kontextgebundenheit der situativen Geschlechterkonstruktionen sichtbar. Denn die Rekonstruktionen sind v.a. hinsichtlich der Rolle als Fachkraft in der Institution Kindertageseinrichtung erklärbar. Hier wird der Einfluss des institutionellen Rahmens im Sinne einer gendered institution (vgl. Acker 1992) bzw. als gendered occupation (Murray 1996) sichtbar.

Sowohl die rekonstruierte Unschuldsvermutung als auch der Generalverdacht verweisen exemplarisch auf die Wirkmächtigkeit kollektivierender Geschlechtszuschreibungen im Alltag der Kindertageseinrichtungen. Bei der Unschuldsvermutung wirken Zuschreibungen im Sinne deduktiver Schlüsse, indem vom Allgemeinen auf den Einzelfall geschlossen wird: Da Weiblichkeit im Hinblick auf niedere Motive körperlicher Annäherungen an Kinder vermeintlich generell nicht bedenklich ist, ist es auch der Einzelfall (hier: die weibliche Forscherin) nicht. Im Hinblick auf den Generalverdacht wirken hingegen induktive Schlüsse: einzelne männliche Täter führen dazu, dass Männlichkeit generell unter Verdacht steht. Beide Schlussfolgerungen gehen mit unterschiedlichen Schwierigkeiten einher, zeigen aber jeweils die Reichweite sozialer Zuschreibungsprozesse im Hinblick auf die Geschlechtszugehörigkeit pädagogischer Fachkräfte.

Hier stellt die viel kritisierte Folie der Zweigeschlechtlichkeit folglich den Dreh- und Angelpunkt der Diskussionen dar (vgl. Fegter 2013, Kubandt 2016). Karsten (2017) fordert jedoch zu Recht, dass ,[e]ntgegen der [...] fortdauernden, häufig vorrangig dualen Konstruktion der Geschlechterrollen von Mädchen und Jungen, Frauen und Männern, Müttern und Vätern“ eine differenziertere Perspektive einzunehmen ist, die „komplexe Möglichkeiten anstelle von polaren Sichtweisen“ bietet (ebd.: 495). Andernfalls sind Kindertageseinrichtungen Orte, in denen bestehende Geschlechterverhältnisse reproduziert und manifestiert werden.

Alles in allem vermischen sich aktuell unterschiedliche Relevanzebenen, wenn Geschlecht fokussiert wird. Zwar macht es durchaus Sinn die personenbezogenen Dienstleistungsberufe und den Beruf der Erzieher*in als Strukturkategorie mit Weiblichkeit zu markieren, um auf ein spezifisches Missverhältnis aufmerksam zu machen. Die jeweiligen, pauschalen, kollektivierenden Positiv- bzw. Negativmarkierungen von Männlich- oder Weiblichkeit scheinen jedoch gerade dann problematisch, wenn die Geschlechtszugehörigkeit nicht nur strukturell in den Fokus gerät, sondern auch zur pädagogisch bedeutsamen Kategorie deklariert wird. Denn indem eine Relevanz unterschiedlicher geschlechtlicher Vorbilder für die Entwicklung von Kindern argumentiert wird, wird Geschlecht nicht nur zum relevanten Faktor einer Gleichstellung auf struktureller und institutioneller Ebene, sondern zu einem pädagogisch bedeutsamen Kriterium für professionelle Praxis und 
Qualität in Kindertageseinrichtungen stilisiert, was deutlicher zu trennen und auch weiter zu hinterfragen wäre.

\section{Literatur}

Acker, Joan (1992): Gendered Institutions. From Sex Roles to Gendered Institutions. In: Contemporary Sociology, 21, 5, S. 565-569.

Aigner, Joseph C./Rohrmann, Tim (2012): Elementar - Männer in der pädagogischen Arbeit. Opladen: Verlag Barbara Budrich.

Brandes, Holger/Andrä, Markus/Röseler, Wenke/Schneider-Andrich, Petra (2016): Macht das Geschlecht einen Unterschied? Ergebnisse der „Tandem- Studie ${ }^{e e}$ zu professionellem Erziehungsverhalten von Frauen und Männern. Opladen: Verlag Barbara Budrich.

Breitenbach, Eva/Bürmann, Ilse/Thünemann, Silvia/Haarmann, Linda (2015): Mehr Männer in Kindertageseinrichtungen. Eine rekonstruktive Studie über Geschlecht und Professionalität. Opladen: Verlag Barbara Budrich.

Buschmeyer, Anna (2013): Zwischen Verdacht und Vorbild. Wie Männer im Erzieherberuf Männlichkeit konstruieren. Wiesbaden: Springer Fachmedien.

Cremers, Michael/Krabel, Jens (2012a): Männer in Kitas. Aktueller Forschungsstand in Deutschland. In: Cremers, Michael/Höyng, Stephan/Krabel, Jens/Rohrmann, Tim (Hrsg.): Männer in Kitas. Opladen: Verlag Barbara Budrich, S. 131-150.

Cremers, Michael/Krabel, Jens (2012b): Generalverdacht und sexueller Missbrauch in Kitas. Bestandsanalyse und Bausteine für ein Schutzkonzept. In: Cremers, Michael/Höyng, Stephan/Krabel, Jens/Rohrmann, Tim (Hrsg.): Männer in Kitas. Opladen: Verlag Barbara Budrich, S. 265-288.

Cremers, Michael/Höyng, Stephan/Krabel, Jens/Rohrmann, Tim (Hrsg.) (2012): Männer in Kitas. Opladen: Verlag Barbara Budrich.

Ehrenspeck, Yvonne (2009): Geschlechterdifferenz, Geschlechterpolarität und „Geistige Mütterlichkeit" - systemtheoretisch betrachtet. In: Glaser, Edith/Andresen, Sabine (Hrsg.): Disziplingeschichte der Erziehungswissenschaft als Geschlechtergeschichte. Jahrbuch Frauen- und Geschlechterforschung in der Erziehungswissenschaft 5. Opladen: Verlag Barbara Budrich, S. 29-47.

Fegter, Susann (2013): Mehr Männer in die Soziale Arbeit?! Neuordnungen von Profession und Geschlecht im aktuellen (fach-)öffentlichen Diskurs. In: Sabla, Kim-Patrick/Plößer, Melanie (Hrsg.): Gendertheorien und Theorien Sozialer Arbeit. Bezüge, Lücken und Herausforderungen. Opladen: Verlag Barbara Budrich, S. $145-162$.

Garfinkel, Harold (1967): Studies in Ethnomethodology. New Jersey: Englewood Cliffs.

Heite, Catrin (2009): Soziale Arbeit als Profession im Kontext geschlechterhierarchischer Positionierungen. In: Glaser, Edith/Andresen, Sabine (Hrsg.): Disziplingeschichte der Erziehungswissenschaft als Geschlechtergeschichte. Jahrbuch Frauen- und Geschlechterforschung in der Erziehungswissenschaft 5. Opladen: Verlag Barbara Budrich, S. 49-59. 
Hoffmann, Hilmar (2012): Erzieherin. In: Horn, Klaus-Peter/Kemnitz, Heidemarie/Marotzki, Winfried/Sandfuchs, Uwe (Hrsg.): Klinkhardt Lexikon Erziehungswissenschaft. Band I. Bad Heilbrunn: Klinkhardt, S. 336-338.

Karsten, Maria-Eleonora (2017): Gender-Mainstreaming in der Sozialpädagogik. In: Otto, Hans-Uwe/Thiersch, Hans (Hrsg.): Handbuch Soziale Arbeit: Grundlagen der Sozialarbeit und Sozialpädagogik. München: Reinhardt Verlag, S. 492-498. [Im Erscheinen]

Kruse, Jan (2014): Qualitative Interviewforschung. Ein integrativer Ansatz. Weinheim: Beltz/Juventa.

Kubandt, Melanie (2016): Geschlechterdifferenzierung in der Kindertageseinrichtung - eine qualitativ-rekonstruktive Studie. Reihe „Studien zu Differenz, Bildung und Kultur". Opladen: Verlag Barbara Budrich.

May, Michael/Rose, Lotte (Hrsg.) (2014): Mehr Männer in die Soziale Arbeit!?. Kontroversen, Konflikte und Konkurrenzen. Opladen: Verlag Barbara Budrich.

Metzinger, Adalbert (2013): Geschichte der Erzieherinnenausbildung als Frauenberuf. In: Fried, Lilian/Roux, Susanna (Hrsg.): Handbuch Pädagogik der frühen Kindheit. Berlin: Cornelsen, S. 390-400.

Murray, Susan B. (1996): "We all love Charles". Men in Child Care and the Social Construction of Gender. In: Gender \& Society 10, 4, S. 368-385.

Rohrmann, Tim/Cremers, Michael/Krabel, Jens (2010): Männer in Kitas - welche Bedeutung hat das Geschlecht pädagogischer Fachkräfte? In: Archiv für Wissenschaft und Praxis der sozialen Arbeit 41, 2, S. 1-12.

Rose, Lotte/Stibane, Friederike (2013): Männliche Fachkräfte und Väter in Kitas. Eine Analyse der Debatte und Projektpraxis. Wiff Expertise. München: DJI.

Rose, Lotte (2012): Wiederkehr von Yin und Yang? Versteckte Geschlechterpolarisierungen im Ruf nach mehr Männern in Erziehung und Bildung. Betrifft Mädchen, 25, 1, S. 10-15.

Strauss, Anselm/Corbin, Juliet (1996): Grounded Theory. Grundlagen Qualitativer Sozialforschung. Weinheim: Beltz. 